\title{
Optimum Energy Control of a Robotic Electric Vehicle at Time with Improved Control Assignment Approaches
}

\author{
Damodara Reddy Ka , Dr. Mukesh Tiwarib \\ a Research Scholar, Dept. of Electrical Engineering, \\ Sri Satya Sai University of Technology \& Medical Sciences, Sehore, Bhopal Indore Road, Madhya Pradesh, India \\ ${ }^{\mathbf{b}}$ Research Guide, Dept. of Electrical Engineering, \\ Sri Satya Sai University of Technology \& Medical Sciences, Sehore, Bhopal Indore Road, Madhya Pradesh, India
}

Article History: Received: 11 January 2021; Accepted: 27 February 2021; Published online: 5 April 2021

\begin{abstract}
Vehicles have made extraordinary commitment to the development of current culture by fulfilling the requirements for more prominent portability in everyday life. The development of Internal Combustion Engine has contributed a ton to the car area. In any case, a lot of harmful discharges as carbon dioxide $\left(\mathrm{CO}_{2}\right)$, carbon monoxide $(\mathrm{CO})$, nitrogen oxides (NOx), unburned hydrocarbons (HCs, etc have been messing contamination up, a dangerous atmospheric devation, and annihilation of the ozone layer. As the current pattern proposes, this method of transport is probably going to supplant inside burning motor (ICE) vehicles soon. Every one of the primary EV segments has various innovations that are at present being used or can get conspicuous later on. Improved control assignment approach strategies are fit for securing ideal force taking care of, obliging framework errors, and fitting ongoing applications can fundamentally improve the powertrain productivity at various working conditions. Rule-based techniques are just organized and effectively implementable continuously; however, a restricted optimality in force dealing with choices can be achieved. Enhancement based strategies are more fit for achieving this optimality at the cost of expanded computational burden. Over the most recent couple of years, these improvement based strategies have been being worked on to suit continuous application utilizing more prescient, recognitive and man-made reasoning apparatuses. This paper presents a conversation about these new patterns continuously improved control assignment approach. Consequently HEVs give better fuel economy contrasted with ICE based vehicles/regular vehicle. Energy management techniques are the calculations that choose the force split among motor constantly to improve the fuel economy and advance the presentation of HEVs.
\end{abstract}

Keywords: Electric Vehicle (EV), Hybrid electric vehicles (HEV), internal combustion engine, Intelligent Transportation.

\section{Introduction}

Relentless natural issues and occasional energy emergencies are significant worries for the car business. Exploring elective powertrain innovations and progressed energy-saving vehicles offers possibly fuel-productive arrangements. Electric Vehicles (EVs) give promising fuel productivity in an examination with ordinary internal combustion engine (ICE)vehicles, because of their ability to recuperate slowing down energy and the way that an extra level of opportunity is accessible to all the more proficiently meet the driver-required force. Their energy management is outlined as force/force split choice, whereby the measure of intensity/force that every one of the rationale sources gives is resolved to fulfill the driver interest, while improving generally vehicular energy productivity. It is usually recognized that upgrades in fuel economy of EVs with decreased emanations is urgently subject to their energy management strategies (EMSs). Several approaches have been proposed to handle the energy management problem, noticing that the presentation of EMSs is firmly identified with numerous variables, e.g., the future speed, the street incline, driver conduct, traffic data. However, the intricacy and vulnerability of driving conditions regularly bargain the presentation of set up EMSs. Instructions to lessen fuel utilization of Hybrid electric vehicles and Plug-in HEVs (PHEVs) establishes a critical exploration subject for elevating a change in outlook to more practical portability.

\section{Electric Vehicle Types}

EVs can run exclusively on electric impetus or they can have an internal combustion engine working close by it. Having just batteries as energy source establishes the fundamental sort of EV, yet there are sorts that can utilize other energy source modes. These can be called hybrid EVs (HEVs). The International Electrotechnical Commission's Technical Committee 69 (Electric Road Vehicles) recommended that vehicles utilizing at least two kinds of energy source, stockpiling or converters can be called as a hybrid electric vehicles as long as in any event one of those give electrical energy. This definition makes a ton of blends workable for hybrid electric vehicles like internal combustion engine and battery, battery and flywheel, battery and capacitor, battery and fuel cell, and so forth Thusly, the normal populace and experts both began calling vehicles with an internal combustion engine and electric engine blend hybrid electric vehicles, battery and capacitor ones as ultracapacitor-helped EVs, and the ones with battery and fuel cell FCEVs. These wordings have gotten broadly acknowledged and as per this standard, EVs can be ordered as follows:

\section{a) Battery Electric Vehicle (BEV)}


EVs with just batteries to give capacity to the drive train are known as BEVs. BEVs need to depend entirely on the energy put away in their battery packs; thusly the scope of such vehicles relies straightforwardly upon the battery limit. Commonly they can cover $100 \mathrm{~km}-250 \mathrm{~km}$ on one charge, though the top-level models can go significantly further, from $300 \mathrm{~km}$ to $500 \mathrm{~km}$. These reaches rely upon driving condition and style, vehicle designs, street conditions, atmosphere, battery type and age. When exhausted, charging the battery pack takes a considerable amount of time contrasted with refueling a traditional ICE vehicle. It can take up to $36 \mathrm{~h}$ totally renew the batteries, there are far less tedious ones too, yet none is equivalent to the brief period needed to top off a fuel tank. Charging time relies upon the charger setup, its foundation and working force level. Favorable circumstances of BEVs are their basic development, activity and accommodation. These don't deliver any ozone depleting substance (GHG), don't make any commotion and hence gainful to the environment.

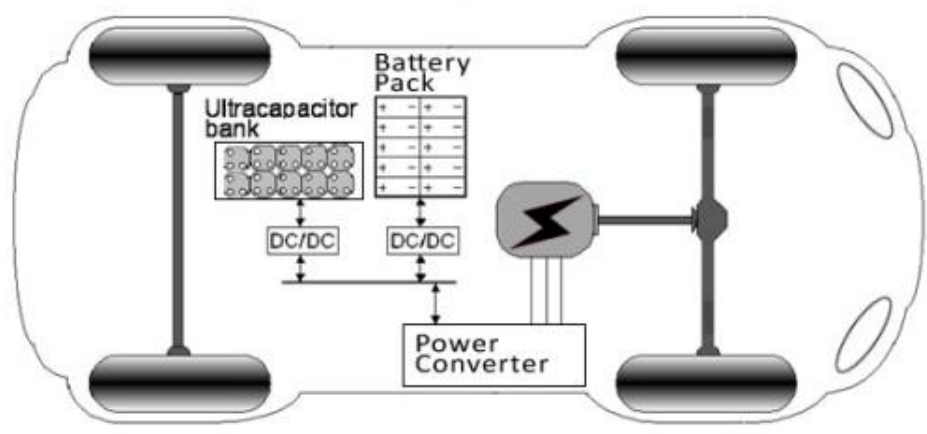

b) Hybrid Electric Vehicle (HEV)

Figure 1 Battery Electric Vehicle

HEVs are grouped chiefly into three classes: (1) series hybrid, (2) parallel hybrid, and (3) series-parallel (powersplit) hybrid. The series setup comprises of an electric engine with an ICE with no mechanical association between them. internal combustion engine is utilized for running a generator when the battery needs more capacity to drive the vehicle; that is, ICE drives an electric generator rather than straightforwardly driving the wheels. Series hybrids have just one drive prepare however require two particular energy transformation measures for all tasks. These two energy transformation measures are fuel to electricity and electricity to drive wheels.

An arrangement hybrid drive train utilizes two force sources which takes care of a solitary force plant (electric engine) that impels the vehicle. An arrangement hybrid electric drive train where: fuel tank is a unidirectional energy source and the internal combustion engine coupled to an electric generator is a unidirectional energy converter. Electrochemical battery pack is the bidirectional energy source and is associated with the force transport by methods for a force gadgets converter (DC/DC converter). Additionally the electric force transport is associated with the controller of the electric footing engine. The foothold engine can be controlled either as an engine (when impels the vehicle) or as generator (to vehicle slowing down). A battery charger can accuse batteries of the energy gave by an electrical organization.

In the equal setup the intensity of the internal combustion engine and the electric engine are added into mechanical coupling and work the drive train by the mechanical transmission. There are distinctive blend of the engine and electric engine power. 1. Force coupling equal hybrid electric drive trains, 2. Speed-coupling equal hybrid electric drive trains, 3 . Force coupling and speed-coupling equal hybrid electric drive trains.

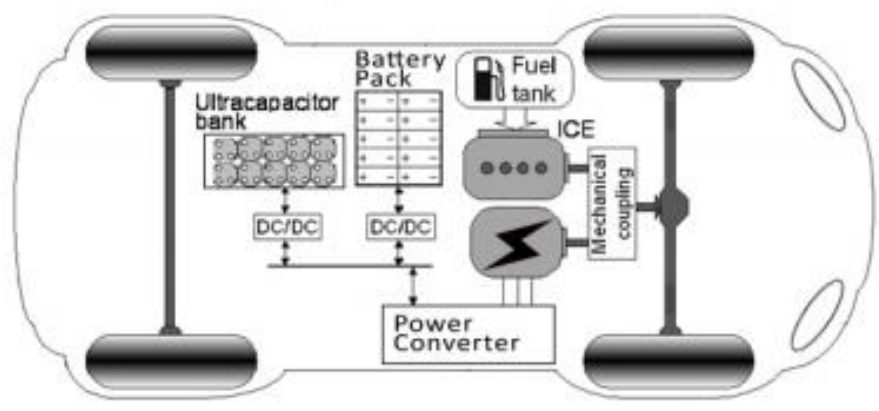

c) Plug-In Hybrid Electric Vehicle (PHEV)

Figure 2Hybrid Electric Vehicle

The PHEV concept emerged to expand the all-electric scope of HEVs. It utilizes both an internal combustion engine and an electrical force train, similar to a HEV, yet the difference between them is that the PHEV utilizes electric impetus as the main driving force, so these vehicles require a greater battery capacity than HEVs. PHEVs start in 'all electric' mode, runs on electricity and when the batteries are low in charge, it calls on the ICE to give a lift or to charge up the battery pack. The internal combustion engine is utilized here to expand the 
reach. PHEVs can charge their batteries directly from the matrix (which HEVs cannot); they additionally have the facility to use regenerative braking. PHEVs' capacity to run exclusively on electricity for more often than not makes its carbon footprint more modest than the HEVs. They consume less fuel also and subsequently reduce the associated cost. The vehicle market is presently very populated with these, Chevrolet Volt and Toyota Prius deals show their popularity as well.

\section{d) Fuel Cell Electric Vehicle (FCEV)}

In addition to these three main sorts, Fuel Cell Electric Vehicle (FCEV) has been introduced to perform significant distances. It utilizes a fuel cell framework to control its on-board electric engine (Fig2.3). Proton Exchange Membrane fuel cells by and large called Polymer Electrolyte Membrane (PEM) fuel cells utilized in FCEVs use hydrogen fuel put away installed and oxygen from the air to deliver electricity. Up to a fuel is provided Fuel cells continue to create electricity, like regular ICEs. However, fuel cells are a lot of cleaner; they convert fuels straightforwardly into electricity by means of an electrochemical cycle that needn't bother with combustion. The created power from a fuel cell stack relies upon the number and size of the individual fuel cells that contain the stack and the surface zone of the Polymer Electrolyte Membrane. A fuel cell vehicle that is fueled with hydrogen produces just water and warmth. By providing perfect, high-productivity, dependable green transportation offices, FCs have become significant innovation in development of electric vehicles.

Also, fuel cells are being developed for transports, boats, cruisers, and numerous different kinds of vehicles. The most recent FCVs to be introduced to the market later one year from now are the all-new Mercedes-Benz BClass F-CELL, Honda FCEV-Concept and Hyundai Tucson-ix35 FCEV.

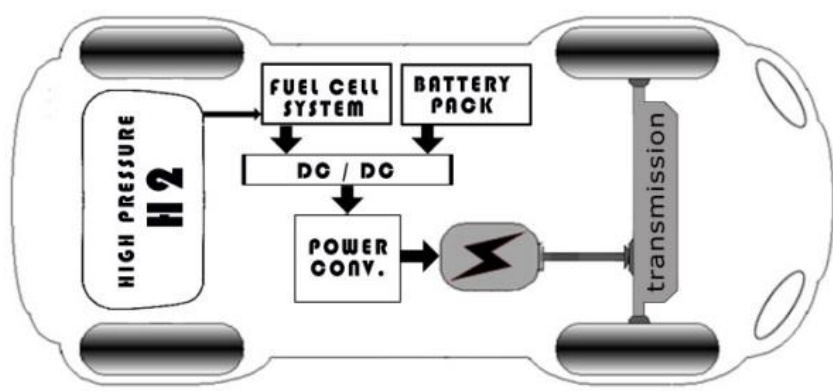

Figure 3Fuel Cell Electric Vehicle

\section{Literature Review}

Zhihan et al (2020):The creator explains that the electric vehicle networks in keen urban communities through enormous information examination innovation, this investigation uses K-implies and fluffy hypothesis in large information investigation innovation to develop a goal work based fluffy mean clustering calculation hypothesis (FCM). At that point, the FCM calculation is improved, and the electric vehicle network is recreated. The outcomes show that in the investigation of organization information transmission execution, when the likelihood of fruitful engendering is $100 \%$ and the $\lambda$ esteem is between $0.01-0.05$, it is nearest to the real outcome, and the information delay is the littlest. In the investigation of the course direction impacts, when facing blocked street segments, the course direction procedure of this examination can restrain the spread of clog viably and achieve convenient evacuation of gridlock. In the further investigation of the effect of various variables on traffic conditions, under course direction, with the increase in market infiltration rate (MPR) of devices, following rate (FR) of vehicles, and blockage level (CL), the improvement of the induction procedure becomes more clear, and more noteworthy financial advantages are achieved. This examination has discovered that utilizing huge information investigation innovation to improve the electric vehicle transportation organizations can decrease the organization information transmission execution delay essentially.

Desheng Li et al (2020): In this paper the creator explains the increase in the utilization of electric vehicles, charging stations may have clog problems. The network energy stockpiling framework can be utilized to fulfill the energy interest for charging electric vehicles batteries. Electric vehicles charging/discharging scheduling for vehicle-to-network and framework to-vehicle activities is challenging on the grounds that clients have distinctive energy prerequisites. Here, a charging and discharging power scheduling calculation settled by an opportunity constrained programming technique was applied to an electric vehicle charging station which contains maximal 500 charging heaps, a $100 \mathrm{~kW} / 500 \mathrm{kWh}$ energy stockpiling framework, and a $400 \mathrm{kWp}$ photovoltaic framework. Accordingly, the force dispatch can be gainful to the charging station and electric vehicle clients under the conceivable effect of the age uncertainty of PV. In light of the proposed calculation, a blueprint for optimizing the agreement limit is dissected for improving the expense of charging stations. The outcomes indicate that the expense of electric vehicles charging can be diminished nearly by half in a certain certainty level of photovoltaic forecasting comparing with uncoordinated strategy.

\section{PROPOSED METHODOLOGY}


Control techniques for hybrid-electric vehicles by and large objective several concurrent goals. The essential one is the minimization of the vehicle fuel utilization, while likewise attempting to minimize emanations and to maintain or improve drivability. Until now, the force management (PM) framework in EVs is fundamentally shaped by two layers; High level programming based oversight and low level equipment based control which can be partitioned into two control layers low level segment and low level control. Both equipment and programming control layers cooperates to streamline PM framework in EV. The numerical detailing of control strategies as far as inputs, yields, targets, and constraints is alluded to as the control Assignment approaches. The arrangement strategy, number of focused goals, and accessibility of inputs (estimated/assessed) influence the appropriateness of these techniques progressively and the optimality of the arrangement. In this specific circumstance, control management techniques are characterized into two main classes: rule-based and enhancement based strategies as delineated in Figure 4.1. In guideline based strategies, the control law is defined by deterministic on-off standards or by fluffy rationale rules. Improvement based techniques execute ideal control approaches to find a worldwide ideal arrangement. This optimality can be obtained using in reverse count in worldwide advancement strategies; however, progressively, instant-wise control choices are taken dependent on assessed future cost minimization.

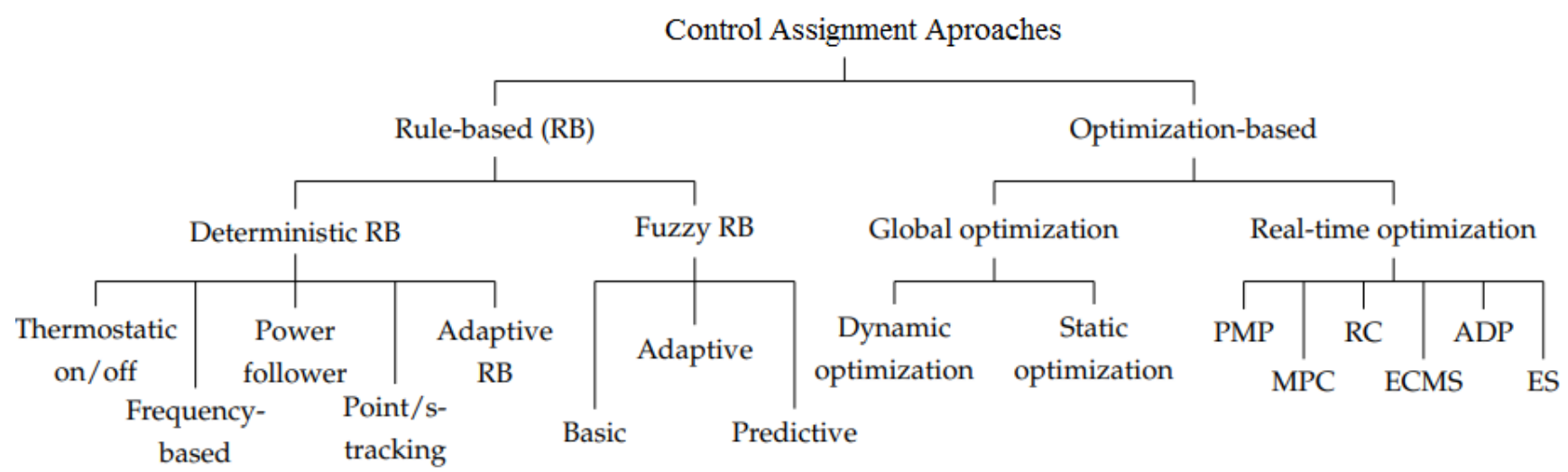

\section{Rule-Based Methods}

Figure 4.1Classification of control assignment approaches

Rule-based control systems are principal control plots that rely upon method of activity. They can be handily actualized with ongoing administrative control to deal with the force stream in a hybrid drive train. The principles are determined dependent on human intelligence, heuristics, or numerical models and by and large without earlier information on a drive cycle.

\section{Deterministic Rule-Based Methods}

Heuristics dependent on examination of intensity stream in a hybrid drive train, productivity/fuel or emanation guides of an ICE, and human encounters are used to plan deterministic standards, for the most part executed through query tables, to spilt mentioned power between power converters.

\section{A. Thermostat (on/off) Control Strategy:}

In this primitive technique, the battery condition of the charge (SOC) is constantly maintained between its preset top and primary concerns by turning ON/OFF the engine. Regardless of its effortlessness, this procedure can't fulfill power requests by the vehicle at all operating conditions. Nevertheless, for an arrangement hybrid electric city transport commuting in prescheduled courses, the indoor regulator control procedure is pertinent.

\section{B. Power Follower (Baseline) Control Strategy:}

This is a notable technique that reformulates the on/off calculations into a further developed at this point appropriate methodology. The main thought is to define distinctive case-based control modules for the force management. In four cases have been set up for engine on/off status dependent on two levels of the battery's condition of charge $\mathrm{SoCb}$ and engine power as outlined in Figure 4.2. 


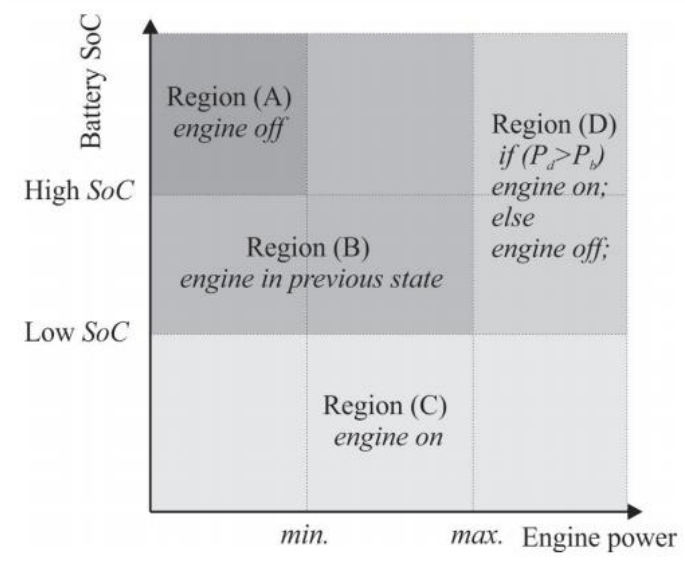

Figure 4.2 Engine on/off rules for power follower strategy

\section{Modified Power Follower-Adaptive RB (ARB)}

In request to improve the adjusted force supporter control assignment, a versatile guideline based energy management technique. The main objective of this methodology is to advance both energy utilization and discharges by introduction of a cost work representing in general fuel utilization and emanations at all up-andcomer operating points. The control methodology utilizes a period found the middle value of speed to find instantaneous energy use and discharge targets.

\section{Frequency-Based Approach}

Using recurrence investigation in force management depends on force request disintegration into high and lowrecurrence segments. Super/ultra-capacitors are described by moderately high force and low energy limit; in this manner, they are more reasonable to assume control over the heap elements. In multi-source HEVs, batteries hold a fair force/energy capacitance and thus are doled out to direct elements load. The low recurrence parts are allotted to sources like engines or fuel cells to relieve the forceful drifters of the heap.

\section{E. Optimal Points Tracking}

This strategy agrees with the baseline approach in that both are targeting ideal engine operating conditions as an essential source; however, these ideal points are here focused at higher accuracy using an endorsed engine map. The utilization of these ideal points is performed at a higher progressive level, at that point the force splitting between optional force sources is directed according to the given accessible force and charging/discharging proficiency of the auxiliary sources.

\section{Fuzzy Rule-Based Methods}

Looking into a hybrid drivetrain as a multi domain, nonlinear, and time-varying plant, fluffy rationale is by all accounts the most coherent way to deal with the problem. Truth be told, instead of using deterministic principles, the dynamic property of fluffy rationale can be received to understand a continuous and imperfect force split. At the end of the day, the fluffy rationale controller is an augmentation of the traditional guideline based controller.

\section{A. Basic Fuzzy}

Effectiveness is chosen dependent on the determination of input, yield, and rule-based control procedure. Two operating modes, in particular, advance fuel use and fluffy productivity modes, are utilized to control drive train activity. The fluffy rationale controller acknowledges battery SOC and the ideal ICE force as inputs. In light of these inputs just as the chose mode, the ICE operating point is set. The force needed by the electric footing engine is the distinction of all out burden power required and power needed from ICE. This control technique utilizes an engine to constrain ICE to work in the area of minimal fuel utilization, while maintaining SOC in battery. Burden balancing is important to fulfill power need and maintain a strategic distance from superfluous charging and discharging of the electrical storage system (ESS).

\section{B. Adaptive Fuzzy}

It is notable that improvement of fuel productivity and emanations are two contending objectives; all in all, an ideal arrangement is consistently a trade off when dealing with effectiveness and different discharges. An ideal operating point can be determined by enhancement of a measure of which weighted fuel (proficiency) and emanations are contending boundaries. Tuning the corresponding loads, the general significance among effectiveness and various emanations can be adjusted because of necessities in different operating conditions. For instance, in territories with basic air contamination, operating points with high outflows are vigorously punished. In another driving circumstance, if the mentioned force by the driver is high, since high efficiencies happen close to high-force areas, it is given the most noteworthy need.

\section{Predictive Fuzzy}


Notwithstanding current driving condition acknowledgment, an expectation of upcoming situations can be integrated into the fluffy controller to achieve close to ideal outcomes. In [26], traffic and street type information are utilized to secure information about not so distant future driving conditions. Moreover, a defensive condition of-wellbeing augmentation procedure is integrated into the technique at the price of moderately higher fuel utilization and emanations.

\section{Optimization-Based Control Strategy}

In improvement based control techniques, the objective of a controller is to minimize the cost work. The cost work (target work) for a HEV may include the discharge, fuel utilization, and force depending on the application. Worldwide ideal arrangements can be obtained by performing improvement over a fixed DC. These control methods don't bring about constant energy management straightforwardly, yet, in view of an instantaneous cost work, an ongoing control procedure can be obtained. This instantaneous cost work depends on the framework factors at the current time as it were. It ought to include comparable fuel utilization to promise self-sustainability of electrical way.

\section{Global Optimization}

There are several announced answers for achieve execution focuses by streamlining of a cost work representing effectiveness and discharges over a drive cycle, yielding to worldwide ideal operating points. These approaches are not material because of their preview nature and computational intricacy. They are, then again, a decent plan device to break down, survey, and change other control assignment approaches.

The use of these strategies can be theoretically classified into dynamic and static enhancement. In powerful streamlining, the control boundaries are definitively determined. The ideal qualities for these boundaries at each time step are looked through using in reverse count to minimize the general cost work. Then again, static streamlining expects to find a fixed ideal incentive for each control boundary that yields adjusted outcomes at different operating conditions.

\section{A. Linear Programming}

The fuel economy improvement is considered as a raised nonlinear streamlining problem, which is finally approximated by linear programming technique. Linear programming is generally utilized for fuel productivity enhancement in arrangement HEVs. Definition of fuel productivity advancement problem using linear programming may bring about a worldwide ideal arrangement. In hybrid force trains, better level of opportunity to control exists. By controlling the stuff proportion and force, an enhanced plan and control of an arrangement hybrid vehicle. The problem is planned as a nonlinear arched advancement problem and approximated as a linear programming problem to find the fuel proficiency. An arched enhancement method for investigation of drive abilities using linear programming, which gives independence from a particular control law. Administrative control assignment for hybrid electric drive trains to minimize fuel utilization. They planned a steady and powerful controller using linear framework inequalities.

\section{B. Dynamic Programming (DP)}

Dynamic programming (DP) was originally utilized in 1940 by Richard Bellman to depict the way toward solving problems where one necessities to find the best choices progressively. DP is both a numerical enhancement technique and a PC programming strategy. In the two settings, it alludes to simplifying a confounded problem by breaking it into more straightforward sub-problems in a recursive way. The very pith of this strategy depends on the principle of optimality. Having a dynamical cycle and the corresponding execution work, there are two different ways to move toward the ideal answer for the problem. One is the Pontryagin's greatest principle and the other is Bellman's dynamic programming. It has the upside of being relevant to both linear and nonlinear frameworks just as constrained and unconstrained problems. In any case, it additionally experiences a severe inconvenience called revile of dimensionality which enhances the computational weight and restricts its application to confounded systems.

Real-Time Optimization

The worldwide enhancement procedures are not straightforwardly application for constant development, considering the way that they are easygoing arrangements. In request to develop a cost work utilized in instantaneous enhancement, notwithstanding a measure for fuel utilization, varieties of the put away electrical energy should likewise be considered to ensure electrical self-sustainability.

\section{A. Equivalent Consumption Minimization Strategy (ECMS)}

ECMS is developed by calculating the total fuel utilization as amount of genuine fuel utilization by ICE and equal fuel utilization of electric engine. This permits a bound together portrayal of both, the energy utilized in the battery and the ICE fuel utilization. Using this methodology, comparable fuel utilization is determined consistently, as an element of the current framework estimated boundaries. No future forecasts are fundamental and a couple of control boundaries are required. These boundaries may shift starting with one HEV geography then onto the next as an element of the driving conditions. ECMS can remunerate the impact of uncertainties of dynamic programming. The solitary drawback of this methodology is that it doesn't ensure charge sustainability of the plant. 


\section{B. Model Predictive Control (MPC)}

Model prescient control (MPC) is a decent strategy for dynamic model of the cycle which is obtained by framework recognizable proof. The main component of the MPC is to permit current timeslot to be streamlined taking future timeslots into account. This is achieved by optimizing a finite time-skyline and implementing the current timeslot as it were. MPC can envision future events and can make control moves accordingly.

\section{Pontryagin's Minimum Principle (PMP)}

The center factor of this strategy is the initial costate determination because of its immediate effect on state direction and arrangement assembly to optimality. Dynamic remedy of this worth is acknowledged by criticism control techniques; for instance, PI-control including likewise spectators to decrease the assessment mistake. In inexact PMP (A-PMP), a straightforward arched estimate of the Hamiltonian could achieve fuel utilization decrease of 6.96\% in a PHEV contrasted with the customary PMP. For an ideal arrangement, PMP gives just fundamental conditions and the adequate conditions are fulfilled by Hamilton-Jacobi-Bellman condition. In PMP, the quantity of nonlinear second-request differential conditions linearly increases with the measurement so the control dependent on PMP sets aside less computational effort for getting an ideal direction yet it very well may be a neighborhood ideal, not a worldwide arrangement. Under certain suppositions ideal direction obtained by PMP should be considered as a worldwide ideal direction. These are as per the following: (1) direction obtained from PMP is extraordinary and fulfills the fundamental and limit conditions, (2) some mathematical properties of the ideal field give the chance of optimality explanation, and (3) as an overall proclamation of the subsequent methodology, the total optimality is, numerically, demonstrated by clear recommendation.

\section{Adaptive Dynamic Programming (ADP)}

Dynamic programming, as a productive enhancement technique, gotten further alterations to suit constant materialness. Here, ADP alludes to the versatile techniques where the main DP calculation is disentangled, discretized, or remade to lessen the computational burden during ongoing control. Intuitively, the streamlined rendition yields a problematic arrangement; however, it beats numerous other ongoing control techniques. The disadvantages of this strategy is that the drive cycle is needed as from the earlier and that little changes in rush hour gridlock or ridership, even for fixed-course transports, can completely change the driving profile.

\section{Conclusion}

Cloud computing technology is increasingly being utilized in enterprises and business markets. In cloud paradigm, a compelling resource allocation strategy is needed for achieving client satisfaction and maximizing the benefit for cloud service suppliers. This paper summarizes the classification of RAS and its impacts in cloud framework. A portion of the strategies talked about above mainly centre around CPU, memory resources, yet are lacking in certain factors. Subsequently this review paper will ideally motivate future researchers to think of smarter and made sure about optimal resource allocation algorithms and framework to fortify the cloud computing paradigm. This paper zeroed in on Hadoop MapReduce resource allocation management methods for multicluster environments. It proposes a novel dynamic space allocation strategy to improve the performance of yarn scheduler and eliminates the load balancing problem. This work demonstrates that the dynamic opening allocation performs in a way that is better than the yarn framework. In future, it is prescribed to concentrate on CPU bandwidth and handling time.

\section{References}

1. Seyedmajid Mousavi, Amir Mosavi, Anna-Maria R. VarkonyiKoczy, Gabor Fazekas, "Dynamic Resource Allocation in Cloud Computing" (2017)

2. Sabbi Vamshi Krishna, Dr. Azad Shrivastava, Dr. Sunil J. Wagh, Dr. T.V. Prasad, "Dynamic Resource Allocation and Job Scheduling To Enhance the Performance of HPC with SDN - A Review" (2020)

3. Pratik P. Pandya, Hitesh A. Bheda, "Dynamic Resource Allocation Techniques in Cloud Computing" (2014)

4. P. Prathap Naidu, K. Raja Sekar, "Cloud Environment: A Review on Dynamic Resource Allocation Schemes" (2018)

5. Ali Belgacem1, Kadda Beghdad-Bey, Hassina Nacer, Sofiane Bouznad, "Efficient dynamic resource allocation method for cloud computing environment" (2020)

6. Chandrasekhar S. Pawar, Rajnikant B. Wagh, "Priority Based Dynamic Resource Allocation in Cloud Computing with Modified Waiting Queue" (2013)

7. Mohit Kumar, S.C. Sharma, "Deadline constrained based dynamic load balancing algorithm with elasticity in cloud environment" (2017)

8. Yong Lu, Na Sun, "An effective task scheduling algorithm based on dynamic energy management and efficient resource utilization in green cloud computing environment" (2017)

9. Zhen Xiao, Weijia Song, and Qi Chen, "Dynamic Resource Allocation using Virtual Machines for Cloud Computing Environment" (2013) 
10. Kong, Zhen, Cheng-ZhongXu, and MinyiGuo. "Mechanism design for stochastic virtual resource allocation in non- cooperative cloud systems." Cloud Computing (CLOUD), 2011 IEEE International Conference on. IEEE, 2011

11. Endo, Patricia Takako, et al. "Resource allocation for distributed cloud: concepts and research challenges." IEEE network25.4 (2011)

12. Minarolli, Dorian, and Bernd Freisleben. "Utility-based resource allocation for virtual machines in cloud computing. "Computers and Communications (ISCC), 2011 IEEE Symposium on. IEEE, 2011 